\title{
Método de Compensación de Acoplamiento Mutuo y Difracción Electromagnética en Arreglos de Antenas de Microtira para GNSS
}

\author{
Marranghelli Ezequiel A. ${ }^{\dagger 1}$, G. Ramón López La Vall ${ }^{\dagger 2}$ and Pedro A. Roncagliolo ${ }^{\dagger 3}$ \\ $\dagger$ Instituto de Investigaciones en Electrónica, Control y Procesamiento de Señales (LEICI) \\ Departamento de Electrotecnia, Facultad de Ingeniería, Universidad Nacional de La Plata (UNLP), La Plata, Argentina. \\ 1 emarranghelli@gmail.com \\ ${ }^{2}$ lopezlavalleding.unlp.edu.ar \\ 3 agustinreing.unlp.edu.ar
}

\begin{abstract}
Resumen-An electromagnetic effects compensation method for antenna array radiation patterns is proposed. Mutual coupling (AM) and electromagnetic diffraction (DE) are main responsible of element radiation patterns distortions on antenna arrays. A linear transformation can be made for undesired effects compensation and differences between radiating elements can be suppressed on some desired direccion of arrivals. This allows simple processing techniques to be employed on later stages of an antenna array. An analysis of a suitable four element plane array is presented. The performance of this method is proved by means of a simulated application example of interference cancellation.
\end{abstract}

Resumen-Se propone un método que permite compensar los efectos electromagnéticos propios de los elementos que conforman un arreglo antenas. El acoplamiento mutuo (AM) y la difracción electromagnética (DE) son principales causantes de distorsiones en los diagramas de radiación de antenas que forman parte de un arreglo. Es posible realizar una transformación lineal que compensa estos efectos indeseados y elimina las diferencias existentes entre elementos radiantes en direcciones de arribo de interés. Esto permite emplear técnicas de procesamiento simples para las etapas posteriores al arreglo en un receptor. Se presenta el analisis de un arreglo plano de cuatro elementos apto para la recepción de señales. Se prueba el desempeño del método por simulación mediante un ejemplo de aplicación de cancelación de interferencia.

Index Terms-Arreglo de Antenas, Acoplamiento Mutuo, Difracción, Patrones de antena, Conformación de Haz Digital.

\section{INTRODUCCIÓN}

Los Sistemas de Navegación Global por Satélite (GNSS) permiten determinar la posición y velocidad de un receptor capaz de sincronizarse con las señales transmitidas por sus satélites. Los GNSS poseen numerosas aplicaciones en diferentes ámbitos, sin embargo en los últimos años su uso civil ha crecido notablemente. De los GNSS existentes en la actualidad, el más difundido y utilizado es el Sistema de Posicionamiento Global (GPS), que se encuentra completamente operativo desde 1994 [1].

Los receptores destinados a aplicaciones GNSS deben ser capaces de recibir señales provenientes de al menos cuatro satélites en simultáneo y en diferentes direcciones del espacio. El sector visible del receptor dependerá de la/s antena/s empleada/s en el mismo y de su orientación. En aplicaciones GNSS de precisión la tendencia es emplear arreglos de ante- nas para mitigar ruido, interferencias, multicamino, así como también aumentar el campo de visión, detectar dirección de arribo, estimar orientación del receptor, etc. Sin embargo, al emplear arreglos de antenas aparecen algunas dificultades y factores adicionales que deben ser tenidos en cuenta, tales como el acomplamiento mútuo (AM), la difracción electromagnética (DE), las variaciones del centro de fase, entre otros. Estos conceptos permiten caracterizar el desempeño de las antenas y su desconocimiento puede resultar en una mayor incertidumbre al realizar la composición de señales recibidas y su posterior procesamiento.

Los arreglos de antenas se diseñan generalmente a partir de un conjunto de antenas preferentemente idénticas y dispuestas entre sí respetando cierta geometría apropiada al objetivo a cumplir. Idealmente, conocer el diagrama de radiación de una de ellas y la posición relativa de las mismas es suficiente para emplear la teoría de arreglos clásica, la cual propone diversos métodos mediante los cuales es posible abstraerse del factor de antena o patrón de radiación y operar simplemente con un factor de arreglo [2].

A pesar de que se logren desarrollar antenas idénticas, el AM y ED se hallan presentes cuando varios elementos radiantes se encuentran próximos entre sí. Estos efectos influyen directamente sobre las características de radiación de las antenas de manera heterogénea, resultando finalmente en antenas con comportamiento dispar [3]. Gracias a que se trata de un problema de naturaleza lineal, es posible obtener soluciones analíticas que describen estos fenómenos.

Se han desarrollado numerosas técnicas que permiten ajustar numéricamente los diagramas de radiación individuales como combinación lineal de patrones ideales idénticos y desacoplados [4] - [5], muchas de ellas con el objetivo de continuar empleando la teoría clásica de arreglos de antenas. Esto consigue minimizar las diferencias en el comportamiento entre antenas, pero las variaciones siguen existiendo. En otros casos, considerando que las variaciones de amplitud y fase no son idénticas para todas las direcciones a causa de la DE, se recurre a realizar un ajuste por mínimos cuadrados para aproximar a un patrón de radiación ideal promedio [6]. En [7] se propone una aproximación que conduce a transformar el comportamiento de un arreglo sobre un determinado sector de interés acotado. El mismo consiste en 
la combinación lineal de patrones individuales que ajusten al comportamiento de un conjunto de antenas isotrópicas ideales. En todos estos casos las variaciones sobre los patrones aproximados resultantes siguen existiendo, lo cual se traduce en variaciones del centro de fase del arreglo, y por lo tanto, del punto geométrico del cual se desea conocer la posición [8].

En aplicaciones en las cuales se dispone de información precisa acerca de la orientación del arreglo de antenas del receptor y la dirección de arribo de las señales, no resulta necesario realizar estas aproximaciones y un conocimiento detallado del diagrama de radiación de cada elemento de un arreglo de antenas es suficiente para cumplir con los mismos objetivos mencionados anteriormente. En arreglos de pocas antenas es posible almacenar esta información y emplearla directamente para combinar apropiadamente las señales recibidas por cada antena sin necesidad de realizar aproximaciones numéricas.

El objetivo de este trabajo es proponer un método sencillo que permita modificar el diagrama de radiación de un arreglo de antenas de microtira para GNSS imponiendo restricciones sólo sobre determinadas direcciones de interés, de manera de compensar el comportamiento de las mismas sin necesidad de recurrir a aproximaciones. Esta compensación puede lograrse cuando la cantidad de direcciones de arribo que se desean normalizar son menores o iguales en número a la cantidad de antenas utilizadas. Por otro lado, en aplicaciones en las cuales sea necesario realizar un procesamiento adaptivo dependiente de estimaciones de dirección de arribo de, por ejemplo posibles interferencias, es posible utilizar el método propuesto en forma iterativa a través de compensaciones sucesivas, a fin de lograr que el arreglo se comporte de manera deseada en la dirección de arribo originalmente desconocida.

El resto del trabajo está organizado de la siguiente forma. En la Sección II se propone la matriz de compensación que modifica la ganancia de antenas en las direcciones de interés. En la Sección III se presenta el diseño de un arreglo plano de cuatro antenas aplicado a la recepción de señales de GPS. La Sección IV está destinada a presentar los resultados de las simulaciones realizadas al arreglo propuesto. En la Sección V se evalúa por simulación el desempeño del método propuesto mediante un ejemplo de aplicación. En la Sección VI se exponen las conclusiones obtenidas.

\section{ESQUEMA DE COMPENSACIÓN}

Consideremos un conjunto de $N$ antenas idénticas dispuestas en un arreglo. El diagrama de radiación de cada antena difiere en relación al de sus elementos vecinos como consecuencia de que el entorno que rodea a cada una de ellas no es el mismo. Por lo tanto, la ganancia de la antena $n$-ésima referida a su propio centro geométrico es $g_{n}(\theta, \phi)$, donde $\theta$ representa elevación y $\phi$ azimuth.

Si evaluamos la amplitud de tensión eléctrica de antena recibida sobre cada una de ellas para un dado instante ' $m$ ', empleando notación fasorial de envolvente compleja y para el caso de $P$ fuentes de señal recibida, el vector de amplitudes puede expresarse como [9]:

$$
\mathbf{x}(m)=\sum_{i=1}^{P} y_{i}(m) \cdot \mathbf{g}\left(\theta_{i}, \phi_{i}\right) \cdot \mathbf{s}\left(\theta_{i}, \phi_{i}\right)+\boldsymbol{e}(m)
$$

donde $y_{i}$ es la amplitud de señal $i$-ésima que arriba de una fuente ubicada en campo lejano en dirección $\left(\theta_{i}, \phi_{i}\right)$ para un conjunto $i=1, \ldots, P ; \mathbf{s}(\theta, \phi)=$ $\left[s_{1}(\theta, \phi), s_{2}(\theta, \phi) \ldots s_{N}(\theta, \phi)\right]^{T}$ es el vector de steering del arreglo para una dada dirección de arribo $(\theta, \phi), \mathbf{g}(\theta, \phi)=$ $\left[g_{1}(\theta, \phi), g_{2}(\theta, \phi) \ldots g_{N}(\theta, \phi)\right]^{T}$ es el vector de ganancias de antena y $\boldsymbol{e}=\left[\begin{array}{llll}e_{1}, & e_{2} & \ldots & e_{N}\end{array}\right]^{T}$ es el vector de ruído presente sobre las impedancias de carga de antena. En notación matricial:

$$
\mathbf{x}(m)=(G \circ S) \mathbf{y}(m)+\boldsymbol{e}(m)
$$

donde $G=\left[\begin{array}{llll}\mathbf{g}\left(\theta_{1}, \phi_{1}\right) & \mathbf{g}\left(\theta_{2}, \phi_{2}\right) & \ldots & \mathbf{g}\left(\theta_{P}, \phi_{P}\right)\end{array}\right], S=$ $\left[\begin{array}{llll}\mathbf{s}\left(\theta_{1}, \phi_{1}\right) & \mathbf{s}\left(\theta_{2}, \phi_{2}\right) & \ldots & \mathbf{s}\left(\theta_{P}, \phi_{P}\right)\end{array}\right], \mathbf{y}=\left[\begin{array}{llll}y_{1} & y_{2} & \ldots & y_{P}\end{array}\right]^{T} \mathrm{y}$ ' $\circ$ ' denota el producto Hadamard.

La ec. (2) representa el vector de amplitudes de antena (envolvente compleja) recibidas y medidas sobre cada canal del receptor. La matriz $[G]_{N \times P}$ de ganancias del arreglo está construida considerando $P$ direcciones de arribo distintas y sobre ella se encuentra almacenado el comportamiento electromagnético que caracteriza al conjunto de antenas empleadas en el entorno en el que realmente se encuentran. Es decir, contiene información de ganancia de campo electromagnético lejano por rayo directo, por DE, AM y rayos reflejados, refractados y difractados por dispersores de campo cercano que hayan sido considerados en el modelo planteado o en la medición de los campos asociados. Por otro lado, la matriz $[S]_{N \times P}$ está formada por la colección de vectores de steering asociados al conjunto de direcciones de arribo de señal, cuyos elementos poseen fase dependiente de la distribución geométrica del arreglo y módulo unitario.

El planteo propuesto a continuación nos permite realizar un pre-procesamiento de las señales adquiridas a través de cada canal a fin de compensar las diferencias en las ganancias complejas de las antenas en estas $\mathrm{P}$ direcciones de arribo. Bajo la suposición de que $P \leq N$ y que las matrices $G$ y $S$ son conocidas, lo que implica conocer en forma precisa el desempeño del arreglo de antenas y las direcciones de arribo de toda onda incidente de interés en un dado instante, es posible realizar esta compensación.

El procedimiento propuesto consiste en eliminar numéricamente el efecto de la matriz $G$ por medio de una matriz de transformación $[W]_{N \times N}$, que produzca un comportamiento equivalente al que podría resultar si se dispusiese de un arreglo de antenas ideales isotrópicas, de ganancia y fase constantes en las $P$ direcciones de interés, es decir $\left[G_{W}\right]=[1]_{N \times P}$. Expresamente, $W$ debe ser tal que:

$W \mathbf{x}=W[(G \circ S) \mathbf{y}+\boldsymbol{e}]=\left(G_{W} \circ S\right) \mathbf{y}+W \boldsymbol{e}=S \mathbf{y}+\tilde{\boldsymbol{e}}=\tilde{\mathbf{x}}$

La solución a este problema es única para el caso $N=P$. En caso de querer emplear el método para $N>P$ debe utilizarse algún criterio para elegir cuál de las posibles soluciones adoptar. Un criterio habitual para este tipo de problemas subdimensionados consiste en la solución de mínima norma y está dada por la matriz pseudoinversa de Moore-Penrose [10]: 


$$
W=S(G \circ S)^{\sharp}
$$

donde $\sharp$ denota la pseudoinversa de Moore-Penrose. Sea $A \in$ $\mathbb{C}^{m \times n}$ de $\operatorname{rango} \operatorname{rank}(A)=r \leq n \leq m$, existen $B \in$ $\mathbb{C}^{m \times r}$ y $C \in \mathbb{C}^{r \times n}$ ambas de rango $r$ tal que $A=B C$. Se define entonces la pseudoinversa de la matriz $A$ como $A^{\sharp}=$ $C^{H}\left(C C^{H}\right)^{-1}\left(B^{H} B\right)^{-1} B^{H}$, con $H$ transpuesta hermítica.

Esto permite desafectar todo fenómeno físico que caracteriza al arreglo de antenas así como la interacción entre sus elementos y con el entorno que lo rodea. De esta forma es posible asumir un comportamiento ideal que simplifica la síntesis del procesamiento posterior. El vector de señales deseadas y queda pesado únicamente por una matriz que sólo depende de la distribución espacial de los elementos del arreglo, es decir, sólo de su geometría.

Por otro lado, el vector de ruído e de $N$ variables aleatorias puede suponerse como ruido aditivo circularmente Gaussiano. En ese caso sus elementos son independientes entre sí y respecto a las señales de interés, e idénticamente distribuidos (i.i.d.). Al aplicar la matriz de transformación la nueva variable aleatoria vectorial de ruido $\tilde{\boldsymbol{e}}$ sigue teniendo distribución normal compleja, pero los canales ya no son independientes entre sí en términos de ruido.

El esquema ilustrado en la Fig. 1 representa el método propuesto. El bloque $W$ debe ser configurado en función de la cantidad de direcciones de arribo de interés y sus respectivas orientaciones.

A pesar de que $W$ pueda hallarse para $P \leq N$ no debe perderse de vista el rango de la matriz resultante $r(W)$. Es decir, a partir de la ec. (4):

$$
r(W) \leq \min \left(r(S), r\left((G \circ S)^{\sharp}\right)\right) \leq N
$$

Por la forma en que se calcula la matriz de compensación, la misma puede no ser de rango completo y en tal caso se logra realizar la compensación, pero también se pierden grados de libertad. Se reduce la cantidad de patrones de antenas equivalentes linealmente independientes respecto de lo que inicialmente se dispone. Para evitar esta situación es conveniente definir $P=N$ direcciones de arribo diferentes ó disponer de los restantes $N-P$ grados de libertad para imponer nuevas restricciones en términos del ruido de antena de manera tal que las matrices $S$ y $(G \circ S)$ sean de rango completo y verificar que el producto $S(G \circ S)^{\sharp}$ también lo sea.

\section{DISEÑO DEL ARREGLO}

Los receptores GNSS normalmente emplean antenas de microtira ya que éstas se caracterizan por ser hemiesféricas, lo cual es importante para maximizar la visibilidad de satélites. Por otra parte, las antenas de microtira tienen la capacidad de polarizarse en forma circular, son de costo reducido, mecánicamente robustas y de bajo perfil. En particular, en este trabajo se utilizan antenas de microtira con patch cuadrado y esquinas truncadas para lograr polarización circular derecha [11]. Las antenas poseen alimentación simple tipo coaxial y utilizan un sustrato de material RT/duroid 6002 [12]. Cabe destacar que la antena propuesta se encuentra adaptada para la banda L1 tanto de GPS como del sistema ruso GLONASS, lo cual le brinda mayor versatilidad al diseño [3]. En la Fig. 2 se presenta el modelo de la antena con sus parámetros más relevantes indicados en la imagen.

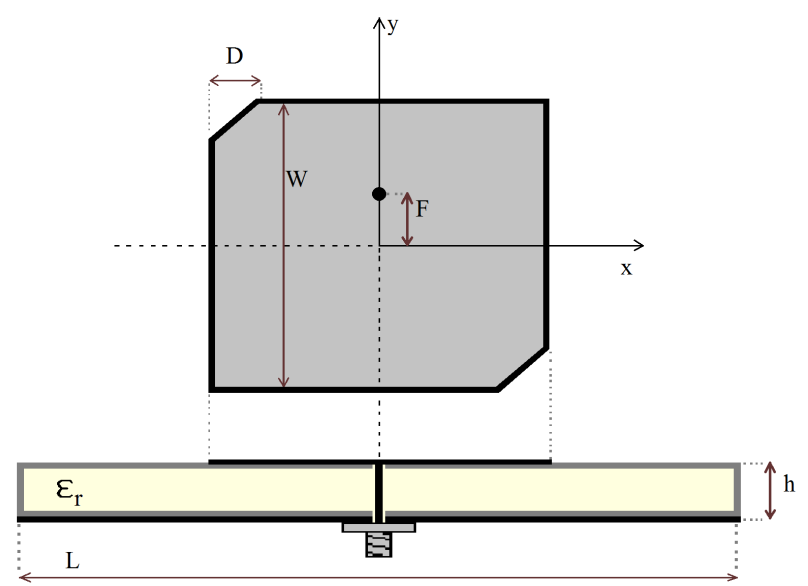

Figura 2. Antena propuesta sintonizada en $1575 \mathrm{MHz}$.

Para implementar el arreglo se dispusieron cuatro antenas alineadas coplanarmente en forma cuadrada y separadas una distancia $d=0,56 \lambda$, como se muestra en la Fig. 3, siendo $\lambda$ la longitud de onda de la frecuencia central de la banda L1 de GPS. La distancia propuesta para la separación entre antenas se debe a la restricción física que imponen las dimensiones de las mismas. Idealmente se utilizaría una separación de $\lambda / 2$ a fin de poder realizar la conformación de haz de manera no ambigua. Con esta separación entre las antenas los efectos de AM y la DE son considerables, lo cual motiva el análisis realizado en este trabajo. Cada antena está construida con un sustrato y un plano de tierra propios, pero a su vez el conjunto se halla montado sobre una superficie metálica que actúa como soporte del arreglo.

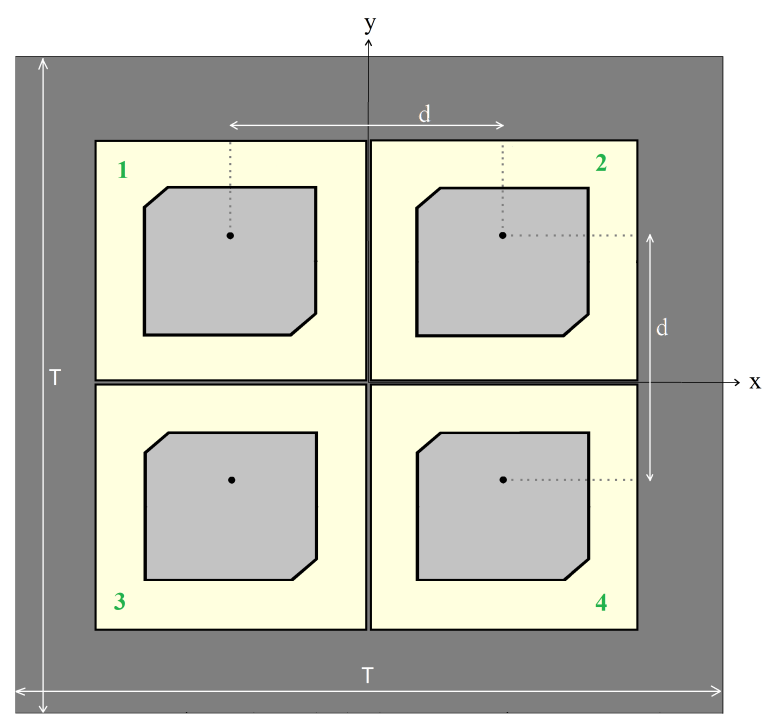

Figura 3. Arreglo $2 \times 2$. 


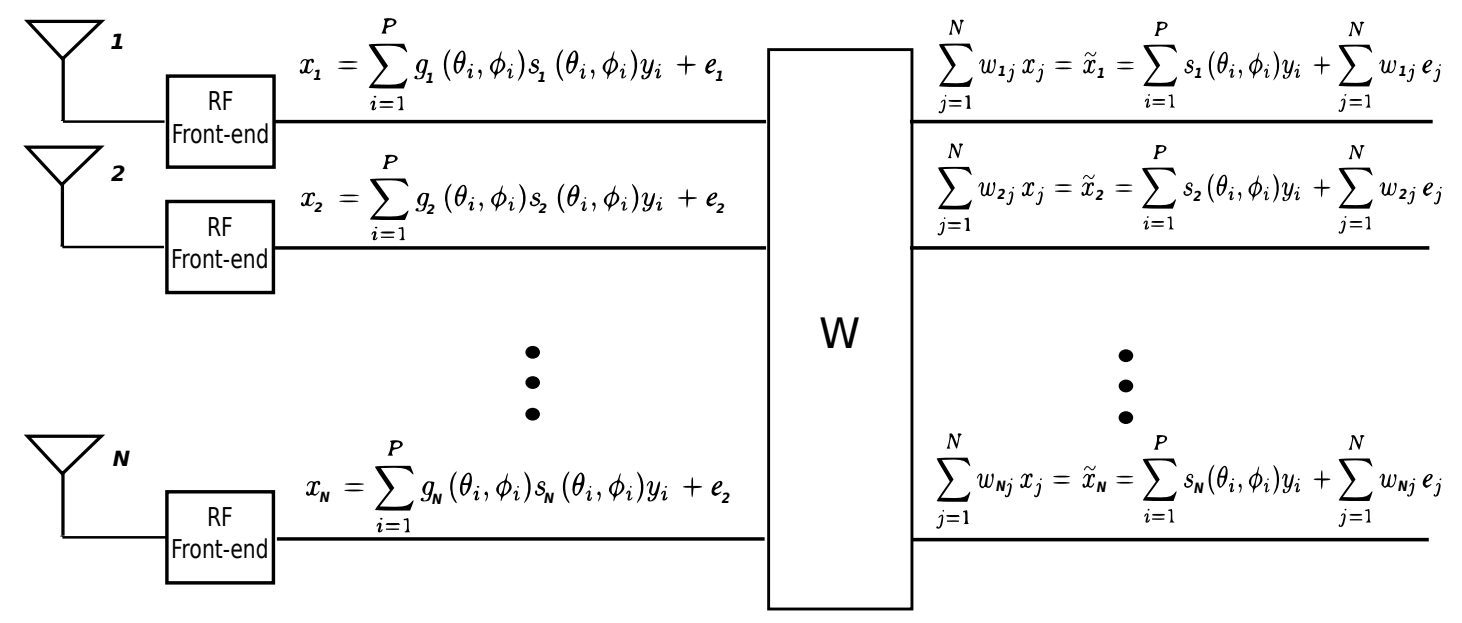

Figura 1. Esquema de compensación propuesto

\section{SIMULACIONES}

Las dimensiones finales de la antena de microtira descrita previamente se obtuvieron por simulación empleando un programa basado en el método de integraciones finitas (FIT) [13], y se resumen en la Tabla I.

Tabla I

DIMENSIONES DE LAS ANTENAS.

\begin{tabular}{|c|c|}
\hline Parámetro & Dimensión $[\mathrm{mm}]$ \\
\hline \hline$D$ & 5,2 \\
\hline$F$ & 10,35 \\
\hline$L$ & 100 \\
\hline$T$ & 600 \\
\hline$W$ & 53,7 \\
\hline$h$ & 1,524 \\
\hline$\varepsilon_{r}$ & 2,94 \\
\hline
\end{tabular}

La Fig. 4 presenta los patrones de radiación obtenidos por simulación cuando cada antena del arreglo de la Fig. 3 se excita mediante una fuente de tensión unitaria a la frecuencia de operación, y el resto de las antenas se encuentran cargadas con la impedancia equivalente de generador. Se ha optado por emplear el sistema de coordenadas Ludwig 3 para facilitar la representación de los diagramas de radiación [14]. Estos diagramas representan la magnitud de ganancia $\left|g_{n}(\theta, \phi)\right|$ de la antena $n$-ésima para todas las direcciones de arribo (y fase $\psi=\angle g_{n}\left(\theta_{i}, \phi_{i}\right)$ en los puntos de interés) antes de realizar el pre-procesamiento. Cada gráfico se presenta en coordenadas polares con radio $\theta$ (Elevación) y orientación $\phi$ (Azimuth). Cuatro direcciones de arribo han sido seleccionadas para evaluar resultados con el método propuesto $(P=N)$. Las mismas son $\left(\theta_{1}, \phi_{1}\right)=\left(10^{\circ}, 45^{\circ}\right)$, $\left(\theta_{2}, \phi_{2}\right)=\left(20^{\circ}, 135^{\circ}\right),\left(\theta_{3}, \phi_{3}\right)=\left(30^{\circ}, 225^{\circ}\right)$ y $\left(\theta_{4}, \phi_{4}\right)=$ $\left(40^{\circ}, 315^{\circ}\right)$.

Por otra parte, en la Fig. 5 se observan los diagramas resultantes luego de aplicar la matriz de transformación $W$ indicada anteriormente. Los diagramas de ganancia que podemos observar son $\left|g_{W, n}(\theta, \phi)\right|$ y fase $\psi=\angle g_{W, n}\left(\theta_{i}, \phi_{i}\right)$ sobre los puntos seleccionados. Los resultados se corresponden con el comportamiento de un arreglo de cuatro antenas idealmente hemisféricas sobre cuatro puntos de interés. Es decir, módulo (normalizado a $3 d B$ ) y fase (normalizada a $0^{\circ}$ ) constantes para cada antena y para cada dirección de arribo sobre los cuatro puntos seleccionados.

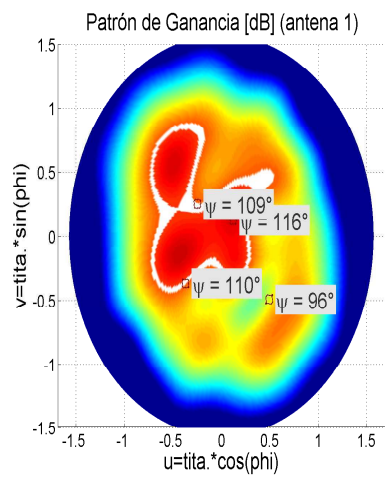

(a)

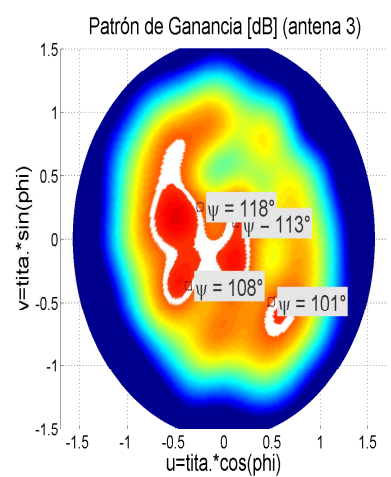

(c)

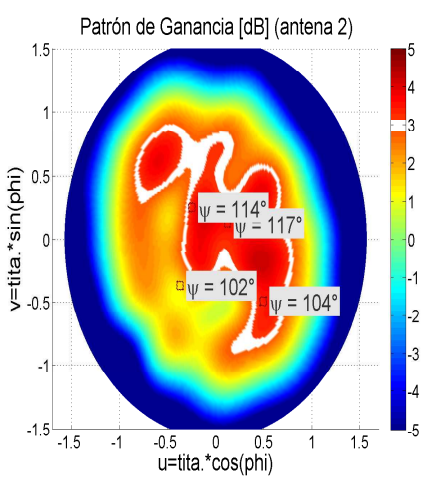

(b)

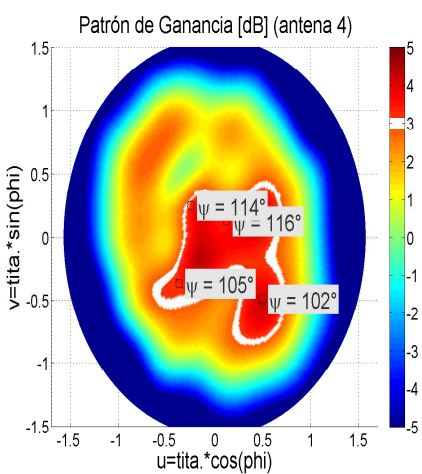

(d)
Figura 4. Patrones sin compensar de antenas (a) 1, (b) 2, (c) 3, (d) 4

Dado que ha sido posible normalizar el comporamiento de los elementos radiantes, ya no será necesario considerar ganancia de antena en las etapas próximas de procesamiento sobre los puntos indicados.

\section{EJEMPLO DE APLICACIÓN}

Considerando los resultados previos es apropiado ahora evaluar el desempeño del arreglo cuando las diferencias entre sus elementos han sido compensadas y comparar resultados. Una de las grandes virtudes de emplear un arreglo de antenas es la posibilidad de conformar un nuevo diagrama de radiación por combinación lineal de los patrones individuales propios de cada elemento. Cuando se desea 


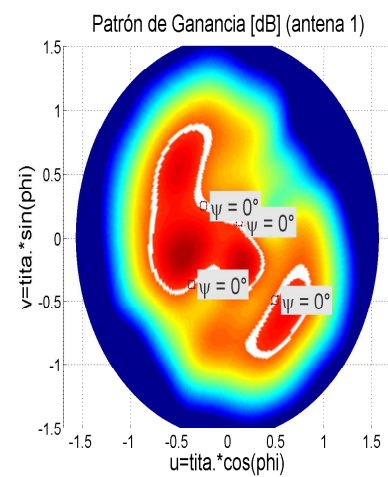

(a)

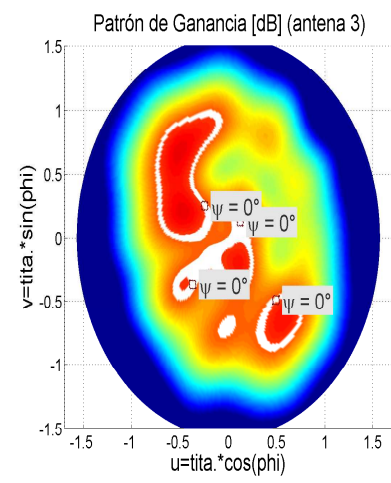

(c)

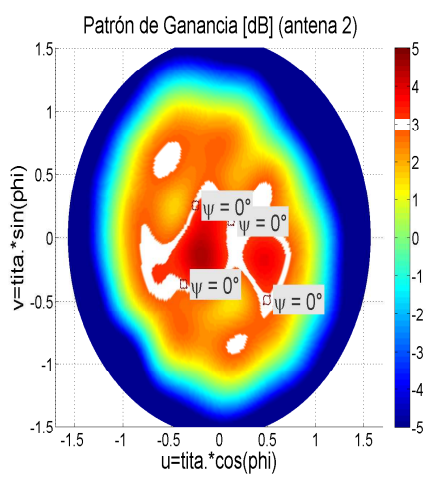

(b)

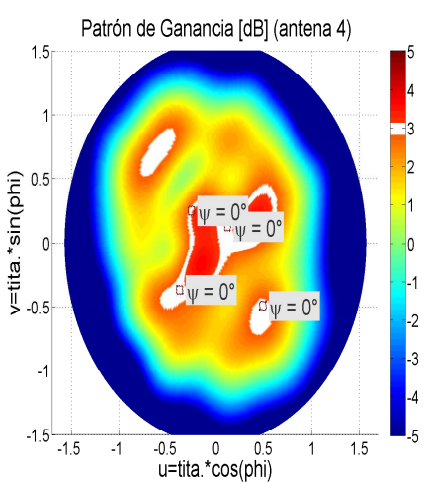

(d)
Figura 5. Patrones compensados de antenas (a) 1, (b) 2, (c) 3, (d) 4

ubicar un punto de máxima ganancia en este nuevo diagrama la mayoria de las técnicas tradicionales de conformación de haz logran buenos resultados sin aplicar correcciones en arreglos de pocos elementos [3]. Sin embargo, existen grandes dificultades al conformar un haz de radiación que contenga un punto o región de ganancia nula tal que logre rechazar por completo (o minimizar lo suficiente) una onda electromagnética interferente que incide sobre el arreglo. Analizaremos este último caso como ejemplo para probar el método propuesto.

$\mathrm{Si}$ consideramos el caso ideal en que disponemos de un arreglo de $N=4$ elementos idénticos, sin tener en cuenta distorsiones causadas por AM y DE, sería posible asignar a los puntos del diagrama $\left(\theta_{i}, \phi_{i}\right), \quad i=1,2,3,4$; la ganancia $G_{i d e a l}\left(\theta_{i}, \phi_{i}\right)$ apropiada simplemente ajustando los coeficientes de peso $a_{n}$ del arreglo dado que la ganancia de los elementos individuales $g_{\text {ideal }}(\theta, \phi)$ es factor común de la ecuación para todo ángulo de arribo:

$$
G_{\text {ideal }}(\theta, \phi)=g_{\text {ideal }}(\theta, \phi) \sum_{n=1}^{N} a_{n} . s_{n}(\theta, \phi)
$$

Conociendo las características reales de los elementos individuales, podemos observar que al intentar colocar un nulo en dirección $\left(\theta_{3}, \phi_{3}\right)=\left(30^{\circ}, 225^{\circ}\right)$ el resultado es el que se puede observar en la Fig. 6 (a), llamado "Sin compensar".

Por otro lado, al emplear el bloque $W$ obtenido mediante la ec. (4) se logran compensar las ganancias individuales de antena $g_{n}\left(\theta_{i}, \phi_{i}\right), i=1,2,3,4$; a módulo $3 d B$ y fase $0^{\circ}$. Es decir, la ganancia compensada $g_{W}\left(\theta_{i}, \phi_{i}\right)$ de los elementos individuales es factor común de la ecuación para las $P$ orientaciones deseadas. Para estos cuatro puntos, los coeficientes obtenidos en base al caso ideal son apropiados para conseguir el funcionamiento requerido ya que el desempeño final del arreglo resulta similar al indicado en la ec. (6):

$$
G_{W}\left(\theta_{i}, \phi_{i}\right)=g_{W}\left(\theta_{i}, \phi_{i}\right) \sum_{n=1}^{N} a_{n} . s_{n}\left(\theta_{i}, \phi_{i}\right)
$$

Puede observarse que empleando los mismos coeficientes obtenidos anteriormente para conformar un nulo en dirección $\left(\theta_{3}, \phi_{3}\right)$ se obtiene el diagrama de radiación "Compensado" presentado en la Fig. 6 (b).

Los resultados muestran una gran diferencia en la calidad de los resultados obtenidos si no se tiene en cuenta la influencia de efectos electromagnéticos que distorsionan los diagramas de radiación individuales. Además, la técnica propuesta permite compensar el desempeño de un arreglo de antenas, previo a las etapas de procesamiento de un receptor, de manera tal que puedan emplearse métodos simples para realizar la conformación de haz a partir de arreglos de antenas reales.

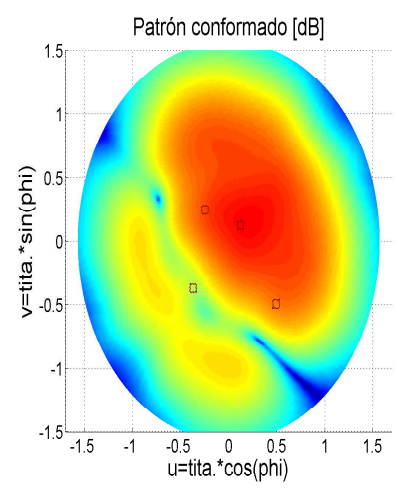

(a) Sin compensar.

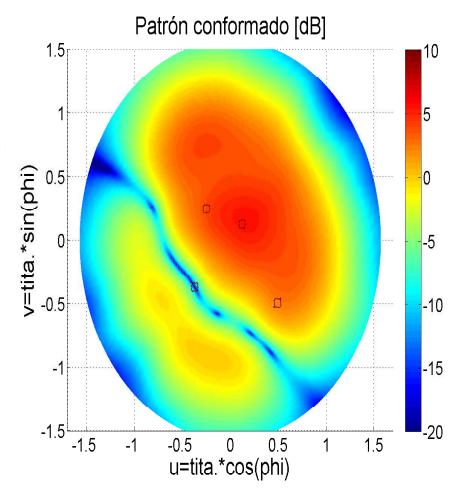

(b) Compensado.
Figura 6. Patrón conformado con nulo en $(\theta, \phi)=\left(30^{\circ}, 225^{\circ}\right)$.

\section{CONCLUSIONES}

En este trabajo se presentó una técnica para compensar los efectos electromagnéticos indeseados presentes en los elementos radiantes que forman un arreglo de antenas. Estos efectos dificultan en ciertos casos el diseño de esquemas de conformación de haz en aplicaciones de precisión.

Mediante un ejemplo ilustrativo ha sido demostrada la necesidad de disponer de información completa del comportamiento de las antenas dispuestas en un arreglo y la posibilidad de emplear métodos de compensación que permitan aprovechar técnicas clásicas de conformación de haz de radiación. El esquema propuesto permite compensar diferencias en el desempeño de elementos radiantes, lo que posibilita el uso de métodos simples para configurar un arreglo de antenas en aplicaciones que requieren gran precisión.

Se analizó un arreglo de cuatro antenas, el cual fue caracterizado en forma numérica mediante simulaciones. Se evaluó el desempeño del mismo en un ejemplo de aplicación para la cancelacion de interferencias, el cual permite apreciar la utilidad del método. Otras técnicas y algoritmos tradicionales de mayor o menor complejidad pueden ser 
empleados en etapas posteriores de procesamiento luego de haber aplicado la matriz de compensación adecuada mediante el método propuesto.

\section{AGRADECIMIENTOS}

Este trabajo fue financiado por ANPCyT PICT 20110909, UNLP Proyecto 11-I-166, CONICET y CIC-PBA.

\section{REFERENCIAS}

[1] Kaplan E. and Hegarty C., Understanding GPS: Principles and Applications, Artech House, 2005.

[2] H. L. Van Trees, Optimum Array Processing. N. York: J. Wiley \& Sons, 2002.

[3] E. Marranghelli, G. R. López La Valle y P. A. Roncagliolo, "Influencia del Acoplamiento Mutuo y Dispersión Electromagnética en Arreglos de Antenas de Microtira para GPS", RPIC XVI, Córdoba, Argentina, 2015.

[4] H. Steyskal and J. S. Herd, "Mutual coupling compensation in small array antennas", IEEE Transactions on Antennas and Propagation, vol. 38, no. 12, pp. 1971-1975, 1990.

[5] S. Henault and M. M. Antar, "Wideband analysis of mutual coupling compensation methods", Int. J. Antennas Propag., Vol. 2011, pp. 124 134, 2011.
[6] M. Zamłyński and P. Słobodzian, "Antenna array radiation pattern modelling which includes mutual coupling and diffraction effects", Proc. 6th European Conf. Antennas Propag. (EuCAP 2012), Praga, 2012, pp. 1627-1631.

[7] T.K. Sarkar, M.C. Wicks, M. Salazar-Palma, R.J. Bonneau, Smart antennas, John Wiley \& Sons, 2005

[8] S.R. Best., "Distance-measurement error associated with antenna phase center displacement in time-reference radio positioning systems", IEEE Antennas and Propagation Magazine, vol. 46, issue 2, pp. 13-22, April 2004.

[9] Daneshmand, S. "GNSS Interference Mitigation Using Antenna Array Processing", PhD Thesis, Department of Geomatics Engineering, University of Calgary, Calgary, Canada, 2013

[10] Strang, G., Linear Algebra and Its Applications, Elsevier Science, 2014.

[11] C. Balanis, Antenna Theory: Analysis and Design, third edition. N. Jersey, USA: J. Wiley \& Sons, 2005.

[12] Rogers Corporation, "RT/duroid 6002 High Frequency Laminates", Data Sheet, 2011.

[13] T. Weiland, "A discretization method for the solution of Maxwell's equations for six component fields: Electronics and Communication", Int. J. Electron. Comun. (AEÜ), Vol. 31, No. 3, pp. 116-120, 1977.

[14] A. C. Ludwig, "The definition of cross polarization", IEEE Trans. Antennas Propag., Vol 21, No. 1, pp 116-119, January 1973. 